\title{
Catalytic Etching of Synthetic Diamond Crystallites by Iron
}

\author{
Tatsuya Ohashi, Wataru Sugimoto, and Yoshio Takasu* \\ Department of Materials and Chemical Engineering, Faculty of Textile Science \\ and Technology, Shinshu University, 3-15-1 Tokida, Ueda 386-8567, Japan \\ E-mail: ytakasu@shinshu-u.ac.jp (Y.Takasu)
}

Keywords: Diamod, Synthetic diamond crystallites, Iron, Gasification, Etching, Catalytic etching

\begin{abstract}
For the expansion of the functionality of diamond crystallites by modification of surface morphology, catalytic etching of synthetic diamond crystallites at $1173 \mathrm{~K}$ by iron, which were loaded by the impregnation method using an aqueous solution of iron nitrate; in a streaming mixed gas $\left(p_{\mathrm{H} 2}=0.1 \mathrm{MPa}, p_{\mathrm{N} 2}=0.9 \mathrm{MPa}\right)$, has been investigated by scanning electron microscopy (SEM) and Raman spectroscopy. The dependence of the crystal planes, $\{111\}$ and $\{100\}$, of the diamond crystallites and the loading amount of iron on the diamond on the etching behavior by iron particles, the morphology of the etch pits, and potential formation of iron carbide through the catalytic etching, were discussed.
\end{abstract}

\section{Introduction}

Carbon materials; such as highly oriented pyrolytic graphite (HOPG), carbon black, activated carbon, diamond and boron-doped diamond (BDD), can be catalytically etched with hydrogen gas at high temperature with the help of various metal nano-particles used as catalysts[1-7]. Such etching behavior by metal particles is called gasification. Gasification can be utilized for the introduction of meso-pores into activated carbon to enhance the functionality of the activated carbon[8-13]; however, gasification has recently been tried to increase the surface roughness of BDD as a pre-treatment for the coating of the oxide catalyst layer, $\operatorname{IrO}_{2}-\mathrm{Ta}_{2} \mathrm{O}_{5}$, on the BDDs $[6,7]$. 
The modification of the surface morphology of diamonds has been reported by many research groups [14-19], Jin el al. focused on the role of the molten metals to etch away the diamond film surface $[14,15]$. The oxidative etching using oxygen, oxygen/water vapor mixture gas and molten potassium nitrate, respectively have been reported [16]. Like steam-activation for the preparation of activated carbon, the surface of BDD could rigorously be roughened by steam-activation treatment [17]. For patterning of diamond, catalytic etching of diamond in the hydrogen atmosphere through thin solid metal film was studied [18]. On the other hand, fine patterning and selective etching of diamond by oxygen plasma etching through aluminum mask were demonstrated [19].

We have previously reported on the catalytic etching of synthetic diamond crystallites by Co [5] and Pt nanoparticles [20]. In both cases, the catalytic etching by those metal particles under a hydrogen atmosphere was highly dependent on the crystal planes of the diamond crystallites. That is, the etched surface of the $\{100\}$ planes by Co particles at $1173 \mathrm{~K}$ consisted of reversed pyramidal-like etch pits with \{111\} walls; however, those on the $\{111\}$ planes conversely showed a characteristic morphology consisting of nano-channels with flat walls perpendicular to the flat $\{111\}$ base. Although a similar phenomenon was observed in the case of the etching by Pt particles, we found that small Pt particles with the unique form of a rectangular parallelepiped-like array were self-embedded in etch pits on the $\{100\}$ planes of synthetic diamond crystallites at $1173 \mathrm{~K}$, while only round shaped Co particles were formed in the case of etching the diamond crystallites by Co particles. The difference in the etching behavior of Co and Pt seems to be affected by either the difference in the solubility of carbon into those metal particles, or by the difference in the melting point of these metals [21]. In our previous study on the catalytic etching of the surface layers of a highly $\{100\}$-oriented diamond coating by Fe, Co, Ni and Pt nanoparticles, only Fe nanoparticles formed a distorted round shape after the etching in a hydrogen atmosphere, probably due to the formation of something like "iron carbide", while the carbon content was not specified [22]. In this study, to expand the functionality of diamond crystallites; e.g., improvement of the abrasion ability, by modification of surface morphology, the etching behavior of diamond crystallites with $\{111\}$ and $\{100\}$ planes by iron was examined. 


\section{Experimental}

The diamond crystallites with a $0.3 \mathrm{~mm}$ average diameter [IMS-25 from Tomei Diamond Co., Ltd.] used were statically produced in $5 \mathrm{GPa}$ at $1573 \mathrm{~K}$. The loading of iron over the diamond crystallites by a impregnation method was conducted using a 0.1 mol dm $\mathrm{dm}^{-3}$ aqueous solution of $\mathrm{Fe}\left(\mathrm{NO}_{3}\right)_{2}$. After $10 \mu \mathrm{L}$ of the precursor solution was added to a beaker in which $10 \mathrm{mg}$ of the synthetic diamond powder was put in, the suspension was dried at $333 \mathrm{~K}$ while stirring. The powder was placed in a Pt boat, which was then placed in a fused silica furnace tube, dried at $333 \mathrm{~K}$ in flowing $\mathrm{N}_{2}$ gas, heated at $5 \mathrm{~K} \mathrm{~min}^{-1}$ to $1123 \mathrm{~K}$ in a flowing gas mixture of $\mathrm{H}_{2}(10 \%)+\mathrm{N}_{2}(90 \%)$, and then retained at the temperature for $2 \mathrm{~h}$. The specimen treated by this procedure was designated as Fe-treated diamond crystallites. A thermogravimetry showed that the decomposition of $\mathrm{Fe}\left(\mathrm{NO}_{3}\right)_{2}$ loaded on the diamond particles was achieved below $573 \mathrm{~K}$, and that the gasification of the diamond with hydrogen and iron particles used as a catalyst began beyond ca. $953 \mathrm{~K}$. The typical loading amount of iron over the diamond powder was 5 mass\%. This loading amount corresponds to ca. $4 \times 10^{4}$ layers of iron, if the iron was coated on the diamond crystallites uniformly. During the heating of the specimen in the hydrogen atmosphere, only methane was detected in the gaseous product by gas-chromatography. The surface structure was examined with a high-resolution scanning electron microscope (HRSEM, Hitachi S-5000) and a Raman spectrometer (Raman Microscope System 3000).

\section{Results and Discussion}

\subsection{Surface morphology of a pristine and the Fe-treated diamond crystallites}

The morphology of the as-received synthetic diamond crystallites was a cubo-octahedral structure with eight $\{111\}$ and six $\{100\}$ planes. Figure 1 shows an SEM image of the typical diamond crystallites. Steps were found on the (100) surface of the diamond; however, no etch pits were observed. Figure 2 shows SEM images of a Fe-treated diamond crystallite, where a (100) plane of the top of the crystallite is surrounded by four $\{111\}$ planes. The comparison of the dark particles in the secondary SEM image of Fig. 2-(a) with roundish and distorted roundish shapes with the white particles in the backscattering SEM image of Fig. 2-(b) strongly suggests that the roundish and distorted roundish particles are iron or iron carbide-like species. Since iron 
forms in various phases with carbon at $1173 \mathrm{~K}, \alpha \mathrm{Fe}$ (bcc) phase in $0 \sim 0.01$ at\% of carbon, $\alpha \mathrm{Fe}+\gamma \mathrm{Fe}$ phase in $0.01 \sim 0.2$ at\% of carbon, $\gamma \mathrm{Fe}$ (fcc) phase in $0.2 \sim 5.4$ at $\%$ of carbon and $\gamma \mathrm{Fe}+\mathrm{Fe}_{3} \mathrm{C}$ in 5.4 25 at\% of carbon [21], we assumed that Fe particles contributed to the etching of the diamond crystallites changed into "iron carbide", although the carbon content could not be specified. The lateral resolution of the energy dispersive X-ray spectrometer (EDX) equipped to the HRSEM adopted to this study was not high enough to determine the composition of the white and dark particles formed on the Fe-treated surfaces. The Fe particles that formed a distorted round shape may be due to the fact that Fe-C binary alloy has the potential to form in various phases with different crystallographic structures at $1173 \mathrm{~K}$. Since uniform coating of the metal over the individual cubo-octahedral diamond crystallite is essentially difficult by the impregnation method adopted to this study, the discussion in this study on the surface modification of the diamond crystallites by iron has to focus on a few parts of the diamond crystallites, especially the typical parts which provide information on the etching by iron particles. To clarify the Fe-treated surface of the diamond, the part indicated with a white rectangle in Fig. 2-(a) was observed at a higher magnification (Fig. 3). This SEM image shows typical characteristic features of the crystal planes, $\{100\}$ and $\{111\}$, which were etched by the iron particles; (1) the extent of etching of the (100) plane was apparently much higher than those of the $\{111\}$ planes, (2) the edge part laying between the $\{111\}$ planes (indicated with a white ellipse in Fig. 3) was rigorously etched. In Fig. 4, a part of the (111) plane [indicated with a black rectangle (A) in Fig 3] was presented at a higher magnification. The secondary electron SEM image of Fig. 4 shows that the (111) surface was etched, having formed various sizes of channels with bases parallel to the (111) plane and with walls perpendicular to the bases. A higher magnification SEM image, shown in Fig. 5, of the part (B) in Fig. 3 clearly shows that channels and etch pits with walls perpendicular to the (111) plane were formed on the (111) surface holding roundish particles of iron or iron carbide-like species. The characteristic feature of the (111) surface is essentially same as that of (A) shown in Fig. 4. Figure 6 presents secondary and back-scattering SEM images of a part of the Fe-treated (111) surface different from the part shown in Fig. 5-(a). The characteristic feature of the (111) surface of the secondary SEM image was essentially 
same as that of Fig. 5-(a). The white particles in the part indicated with a black and a white ellipse in Fig. 6 will be discussed in section 3.2. Secondary and back-scattering SEM images of the Fe-treated (100) surface are presented in Fig. 7. The characteristic large round particles typically indicated with the white rectangles seem to be secondary particles constituted with smaller iron carbide-like species, because very small particles with sub-micrometer in size are observed on the surface of the large particles. The characteristic feature of the back-scattering SEM image is also observed on the surface of the large particles formed on the Fe-treated (100) and (111) surfaces shown in Fig. 2-(b).

Contrary to the etching feature of the $\{111\}$ planes, the (100) surface of this crystallite was severely etched by iron as shown in Fig. 5-(b). In order to clarify the initial stage of the etching process of the $\{100\}$ planes, another diamond crystallite selected from the Fe-treated diamond crystallites, on which a smaller amount of iron might have been loaded than that on the part shown above, was observed by the SEM. An SEM image of the Fe-treated diamond crystallite is shown in Fig. 8, and SEM images, a secondary electron image and a backscattering electron image; of a part of the (100) plane observed at higher magnification, are presented in Fig. 9. The darkish rectangles indicated with (a), (b), (c) and (d) in Fig. 8 are the parts where primary electron beam was irradiated to the surface for the SEM observation. Figure 9 shows the SEM images from the part (a) indicated in Fig. 8. Many etch pits holding distorted roundish particles, which were probably iron or iron carbide-like species, were observed in these SEM images. The etch pit array formed on this crystal plane reflected the arrangement of the carbon atoms of the $\{100\}$ plane of the diamond structure. Although most etch pits held roundish or distorted roundish iron or iron carbide-like particles as indicated with the white circles, a few etch pits held none of such particles as indicated with white squares in Figs. 10-(a) and 10-(b). The formation of the vacant etch pits suggests that the iron nanoparticles could slip out of the etch pits, probably as a melted state, from the etch pits of the diamond crystallites, because the melting point of a metal nanoparticle generally decreases with a decrease in the particle size [23]. However, another probability must also be considered; that is, Yoshida and his co-workers found that iron carbide particles were movable in a solid state and contributed to the formation of carbon nanotubes, even at $873 \mathrm{~K}$ [24]. 


\subsection{Surface state of the Fe-treated diamond crystallites}

As typically shown in the backscattering SEM image of Fig. 4-(b), three kinds of roundish particles were observed on the Fe-treated diamond crystallites, i.e., white particles covered with darkish thick film (in the part indicated with a white rectangle), white particles on darkish particles (in the part indicated with a white ellipse), and darkish particles without white particles (in the part indicated with a white circle). The particles in the part indicated with a black rectangle in the secondary electron SEM image in Fig. 4-(a) seem to be composed of two phases at least, because the back-scattering electron SEM image of the particles shown in Fig. 4-(b) suggests that each white cores of the particles look like to be covered with darkish thick film. Since the brightness of carbide-like species in a backscattering SEM is higher than that of carbon, the cores seem to be iron carbide-like particles and the thick film to be carbon. The carbon film must be formed through the discharge from the respective iron carbide-like particles after the saturation of the carbon which had been incorporated into the carbide-like particles from the diamond. As shown in the parts indicated with ellipses in Figs. 4 and 6, some white particles put on, on-top or side-on, the darkish particles. In the case of the darkish particles, typically shown in the white rectangle, the iron carbide cores seem not to be observed due to the limitation of the escape depth of the reflected electrons in the back-scattering SEM through the thick carbon film.

The surfaces of the pristine and Fe-treated diamond crystallites were also examined by Raman spectroscopy. Figure 10-A shows the Raman spectra of the (a) pristine diamond crystallites and Fe-treated diamond crystallites (b) loading 2 mass\%Fe and (c) 5 mass\%Fe. Figure 10-B shows those specimens which were treated by $0.5 \mathrm{M}$ sulfuric acid at $333 \mathrm{~K}$ to remove iron and iron compounds from the Fe-treated diamond crystallites. All of these Raman spectra show that the diamond structure was retained without the formation of any graphite species by the Fe-treatment.

\section{Conclusion}

Catalytic etching of synthetic diamond crystallites at $1123 \mathrm{~K}$ by iron particles in a stream of a mixed gas of hydrogen and nitrogen, was found to be highly dependent not only on the crystal plane of the diamond crystallites, but also on the loading amount of iron similar to the cases of the etching by Co and Pt. On a (100) plane, where a small 
amount of iron was loaded, an etch pit array holding roundish iron or iron carbide-like particles were formed after the Fe-treatment. The $\{111\}$ planes of the Fe-treated diamond showed a characteristic morphology which consisted of channels with flat walls perpendicular to the flat bases and etch pits with a few flat steps, holding roundish iron or iron carbide-like species on the channels and in the etch pits. The iron particles which contributed to the etching of the diamond crystallites might have changed into iron carbide-like species. Even after the Fe-treatment at $1123 \mathrm{~K}$, the diamond structure was retained without the formation of any graphite species so long as the Raman spectroscopy of the surfaces. No rectangular parallelepiped array of iron or iron compound was observed as different from the case of Pt-treated diamond crystallites. These findings provide useful information for the modification of the surface morphology of diamond crystallites to increase their functionalities.

\section{References}

[1] Y. Takasu, R. Matsuyama, S. Konishi, W. Sugimoto, Y. Murakami, Dependence of the preparation temperature of $\mathrm{Pt}_{0.7} \mathrm{Co}_{0.3} / \mathrm{C}$ catalysts on the structure of the alloy particles and the carbon supports, Electrochem. Solid-State Lett. 8 (2005) B34-B37.

[2] Y. Takasu, S. Konishi, R. Miyoshi, K. Nukii, T. Matsuse, W. Sugimoto, et al., Catalytic linear grooving of graphite surface layers by $\mathrm{Pt}, \mathrm{Ru}$, and PtRu nanoparticles, Chem. Lett. 34 (2005) 1008-1009.

[3] S. Konishi, W. Sugimoto, Y. Murakami, Y. Takasu, Catalytic creation of channels in the surface layers of highly oriented pyrolytic graphite by cobalt nanoparticles, Carbon 44 (2006) 2338-2340.

[4] T. Iwazaki, T. Semba, S. Konishi, T. Sezai, Y. Murakami, W. Sugimoto, et al., Catalytic excavation and graphitization of activated carbon by cobalt nanoparticles, Chem. Lett. 37 (2008) 1194-1195.

[5] S. Konishi, T. Ohashi, W. Sugimoto, Y. Takasu, Effect of the crystal plane on the catalytic etching behavior of diamond crystallites by cobalt nanoparticles, Chem. Lett. 35 (2006) 1216-1217.

[6] Y. Takasu, S. Konishi, W. Sugimoto, Y. Murakami, Catalytic formation of nanochannels in the surface layers of diamonds by metal nanoparticles, Electrochem. Solid-State Lett. 9 (2006) C114-C117. 
[7] T. Ohashi, W. Sugimoto, Y. Takasu, Catalytic roughening of surface layers of BDD for various applications, Electrochim. Acta 54 (2009) 5223-5229.

[8] D.D. Eley, P.W. Selwood, P.B. Weisz, Advances in catalysis XI, Academic Press Inc, New York and London, 1959.

[9] N. R. Khalili, M. Campbell, G. Sandi, J. Golas, Production of micro- and mesoporous activated carbon from paper mill sludge - I. Effect of zinc chloride activation, Carbon 38 (2000) 1905-1915.

[10] A. Ahmadpour, D.D. Do, The preparation of active carbons from coal by chemical and physical activation, Carbon 34 (1996) 471-479.

[11] F. Rodriguezreinoso, M. Molinasabio, M.T. Gonzalez, The use of steam and $\mathrm{CO}_{2}$ as activating agents in the preparation of activated carbons, Carbon 33 (1995) 15-23.

[12] R.T.K. Baker, In situ electron microscopy studies of catalyst particle behavior, Catal. Rev.-Sci. Eng. 19 (1979) 161-209.

[13] Y. Tamai, H. Watanabe, A. Tomita, Catalytic gasification of carbon with steam, carbon dioxide and hydrogen, Carbon 15 (1977) 103-106.

[14] S. Jin, J.E. Graebner, M. McCormack, T.H. Tiefel, A. Katz, W.C. Dautremontsmith, Shaping of diamond films by etching with molten rare-earth-metals, Nature 362 (1993) 822-824.

[15] M. McCormack, S. Jin, J.E. Graebner, T.H. Tiefel, G.W. Kammlott, Low temperature thinning of thick chemically vapor-deposited diamond films with a molten Ce-Ni alloy, Diamond \& Relat. Mater. 3 (1994) 254-258.

[16] F.K. de Theije, E. van Veenendaal, W.J.P. van Enckevort, E. Vlieg, Oxidative etching of cleaved synthetic diamond $\{111\}$ surfaces, Surf. Sci. 492 (2001) 91-105.

[17] T. Ohashi, J. Zhang, Y. Takasu, W. Sugimoto, Steam activation of boron-doped diamond electrodes, Electrochim. Acta 56 (2011) 5599-5604.

[18] V.G. Ralchenko, T.V. Kononenko, S.M. Pimenov, N.V. Chernenko, E.N. Loubnin, V.Y. Armeyev, et al., Catalytic interaction of Fe, Ni and Pt with diamond films: patterning applications, Diamond \& Relat. Mater. 2 (1993) 904-909.

[19] H. Masuda, M. Watanabe, K. Yasui, D. Tryk, T. Rao, A. Fujishima, Fabrication of a nanostructured diamond honeycomb film, Adv. Mater. 12 (2000) 444-447.

[20] T. Ohashi, W. Sugimoto, Y. Takasu, Self-embedment of small rectangular parallelepiped platinum particle array in etch pits on $\{100\}$ planes of diamond 
crystallites, Bull. Chem. Soc. Jpn. 84 (2011) 276-278.

[21] T. B. Massalski, H. Okamoto, P. R. Subramanian, L. Kacprzak, Binary Alloy Phase Diagrams, 2nd ed., ASM International, Ohio, 1990, Vol. 1, pp.835-873.

[22] T. Ohashi, W. Sugimoto, Y. Takasu, Catalytic etching of $\{100\}$-oriented diamond coating with Fe, Co, Ni, and Pt nanoparticles under hydrogen, Diamond \& Relat. Mater. 20(2011) 1165-1170.

[23] P. Buffat, J.P. Borel, Size effect on the melting temperature of gold particles, Phys. Rev. A 13(1976) 2287-2298.

[24] H. Yoshida, S. Takeda, T. Uchiyama, H. Kohno, Y. Homma, Nano Lett. 8 (2008) 2082.

\section{Figure captions}

Fig. 1 An SEM micrograph of the synthetic diamond crystallite.

Fig. 2 SEM micrographs of an Fe-treated diamond crystallite, showing a (100) and four $\{111\}$ planes, prepared by the impregnation method under a gas mixture stream of high-purity $\mathrm{H}_{2}(10 \%)+\mathrm{N}_{2}(90 \%)$ at $1173 \mathrm{~K}$ for $2 \mathrm{~h}$. (a) is a secondary electron image, and (b) is its backscattering electron image.

Fig. 3 An SEM micrograph of an Fe-treated diamond crystallite, showing a corner part of the white square indicated in Fig. 2.

Fig. 4 SEM micrographs of an Fe-treated diamond crystallite, showing a (111) plane indicated with (A) in Fig. 3. (a) is a secondary electron image, and (b) is its backscattering electron image.

Fig. 5 SEM micrographs of an Fe-treated diamond crystallite, showing a (111) plane indicated with (B) and a (100) plane indicated with (C) in Fig. 3.

Fig. 6 SEM micrographs of an Fe-treated diamond crystallite, showing a (111) plane of a different part from Fig. 4. (a) is a secondary electron image, and (b) is its 
backscattering electron image.

Fig. 7 SEM micrographs of an Fe-treated diamond crystallite, showing a (100) plane of a different part from Fig. 4. (a) is a secondary electron image, and (b) is its backscattering electron image.

Fig. 8 An SEM micrograph of an Fe-treated diamond crystallite, showing a (100) and three $\{111\}$ planes.

Fig. 9 SEM micrographs of an Fe-loaded $\{100\}$ surface of the diamond crystallite. (a) is a secondary electron image, and (b) is its backscattering electron image.

Fig. 10 Raman spectra of the pristine diamond crystallites (a) and the Fe-treated diamond crystallites. The loading amount of Fe was (b) 2 mass\%Fe and (c) 5 mass\% Fe. The left spectra, in Fig. 10-A, show the specimens before the acid-treatment, and the right spectra, in Fig. 10-B, show those after the acid-treatment to remove the iron and iron carbide-like species form the Fe-treated diamond crystallites by $0.5 \mathrm{M} \mathrm{H}_{2} \mathrm{SO}_{4}$ at $333 \mathrm{~K}$. 


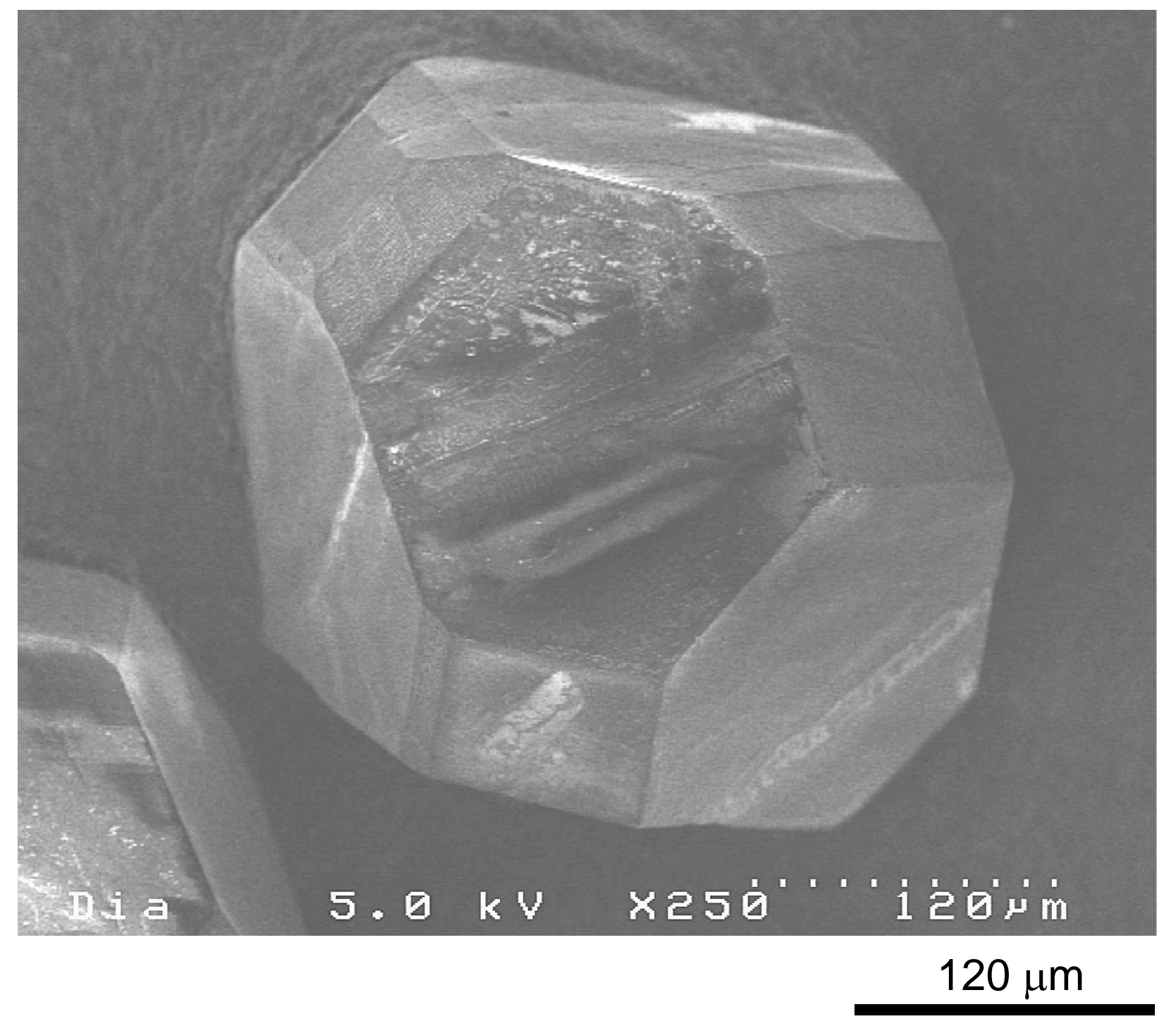

Fig. 1, T. Ohashi et al. 


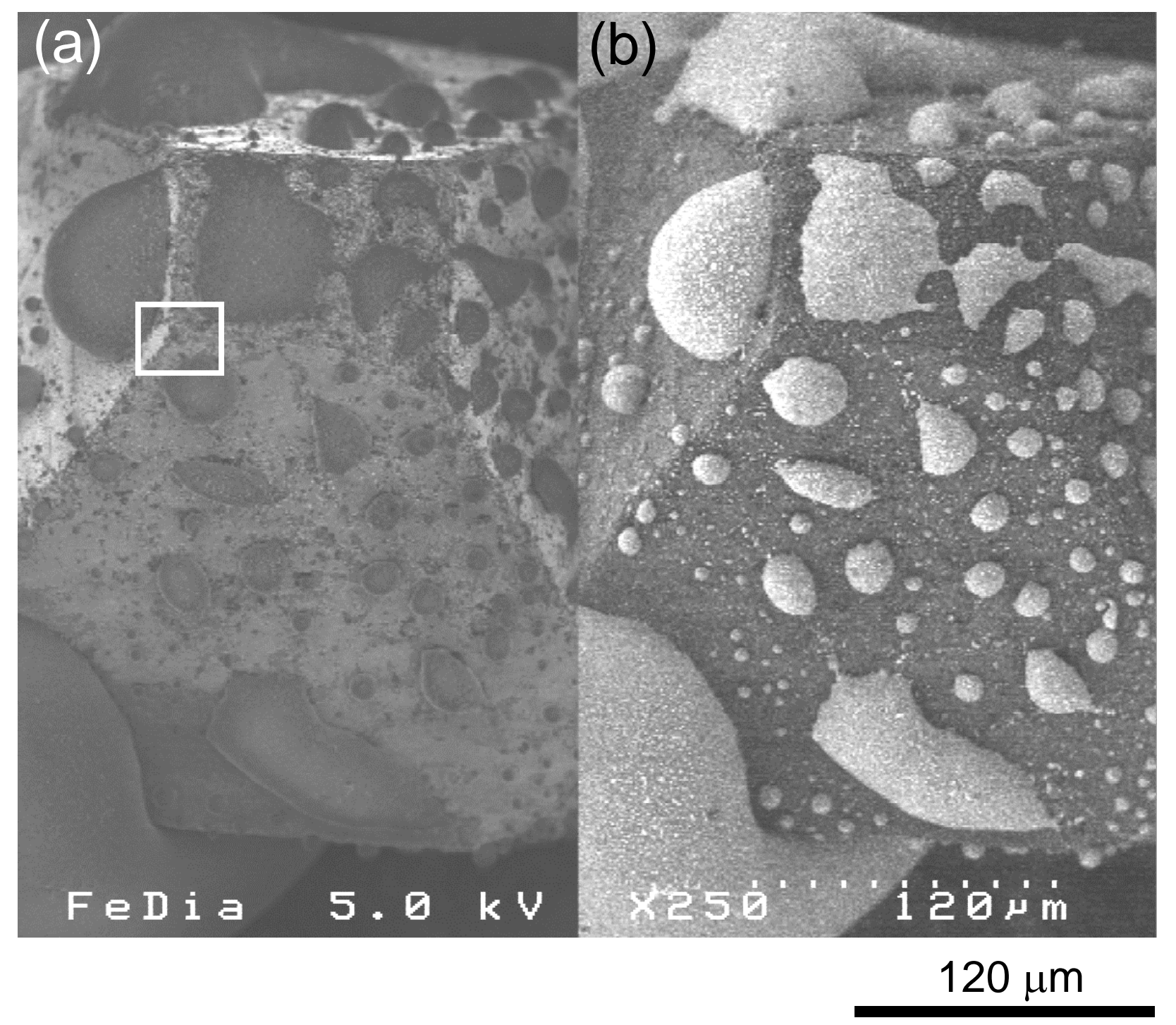

Fig. 2, T. Ohashi et al. 


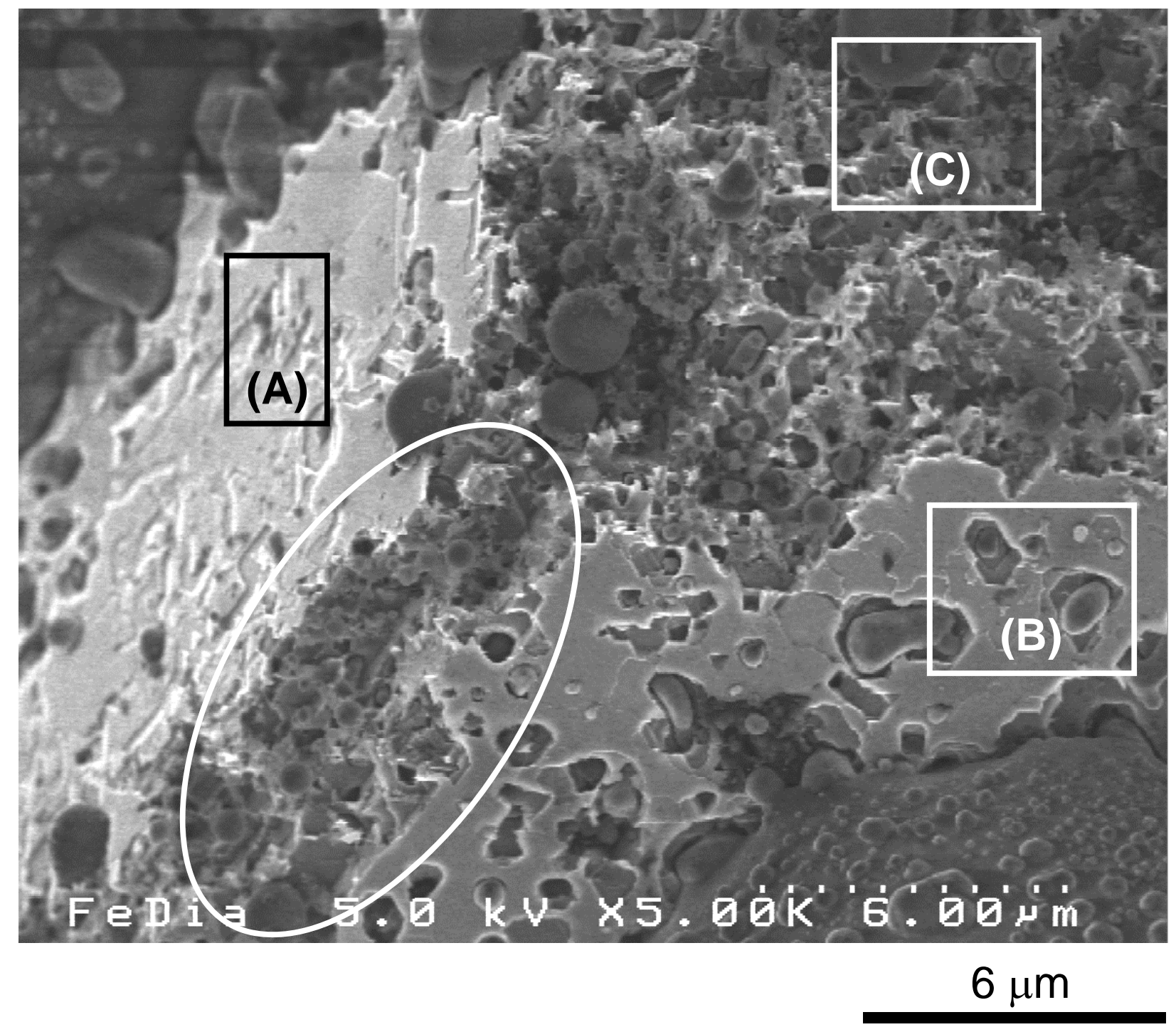

Fig. 3, T. Ohashi et al. 


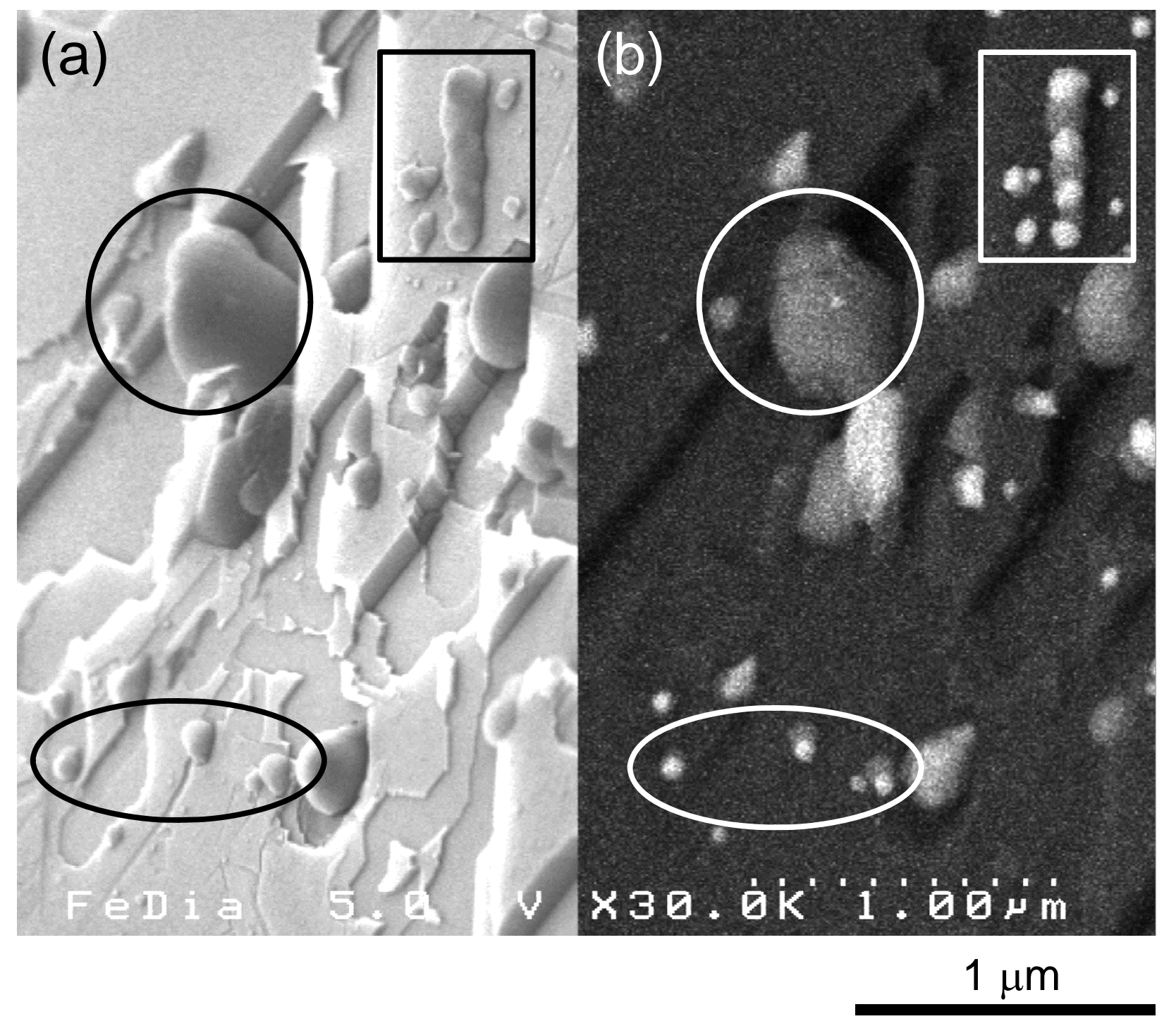

Fig. 4, T. Ohashi et al. 

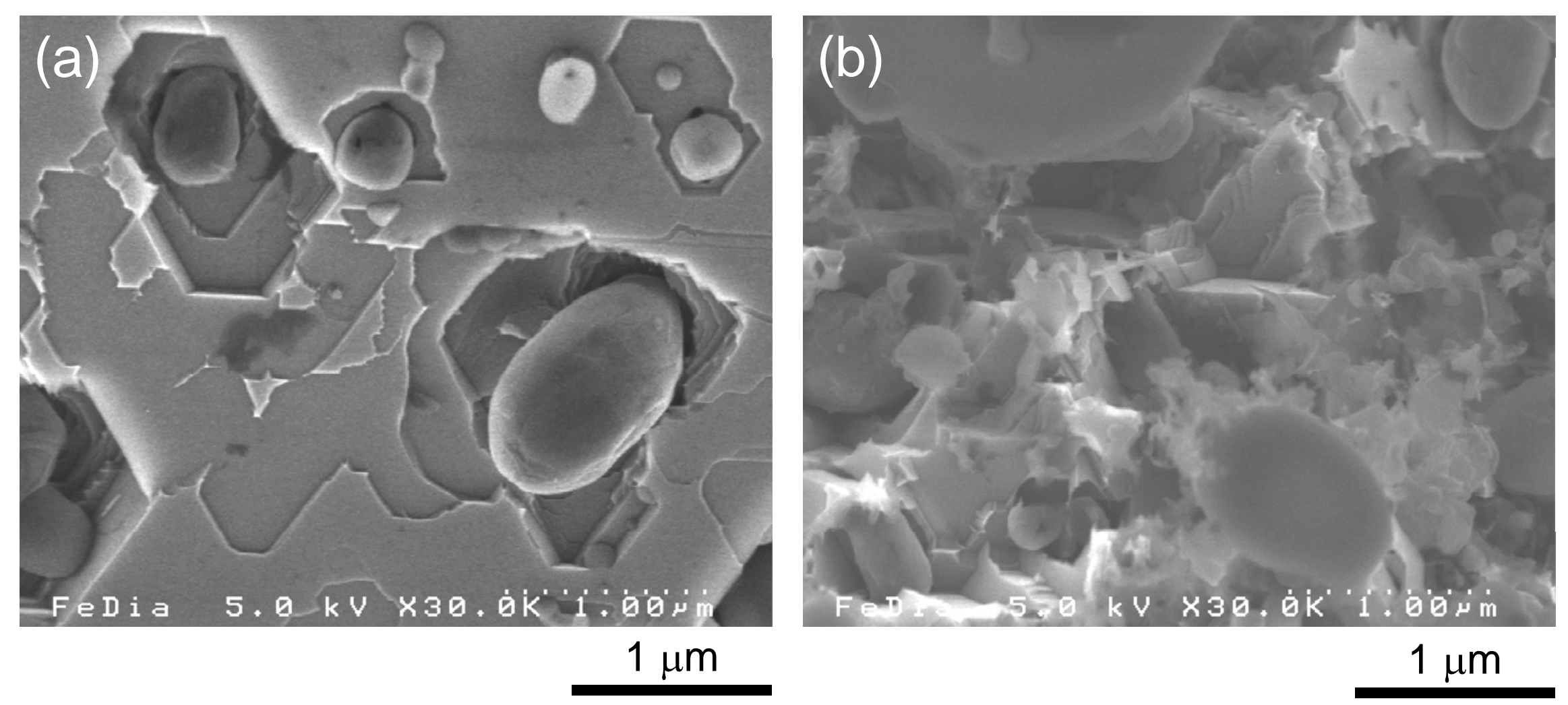

Fig. 5, T. Ohashi et al. 


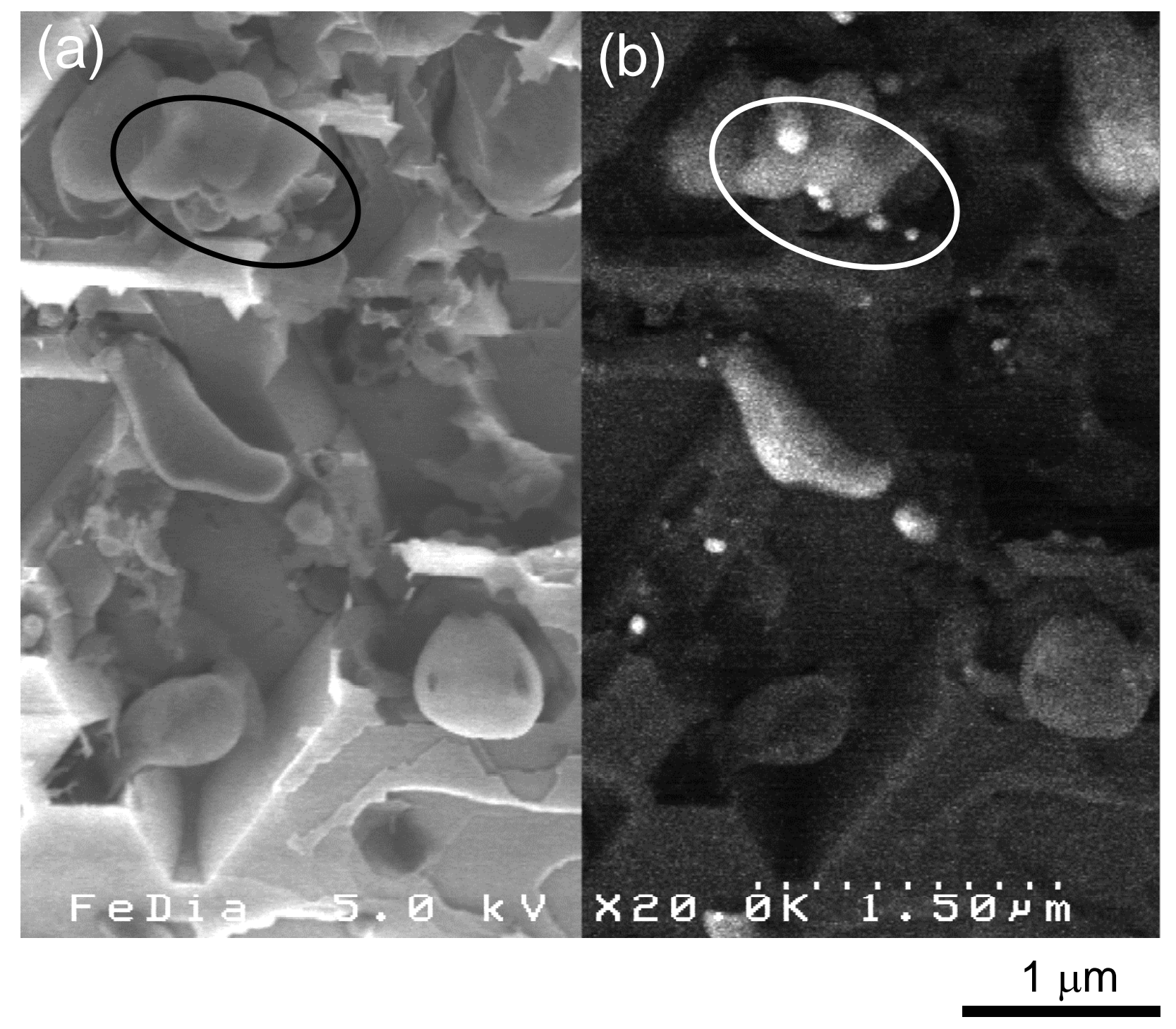

Fig. 6, T. Ohashi et al. 


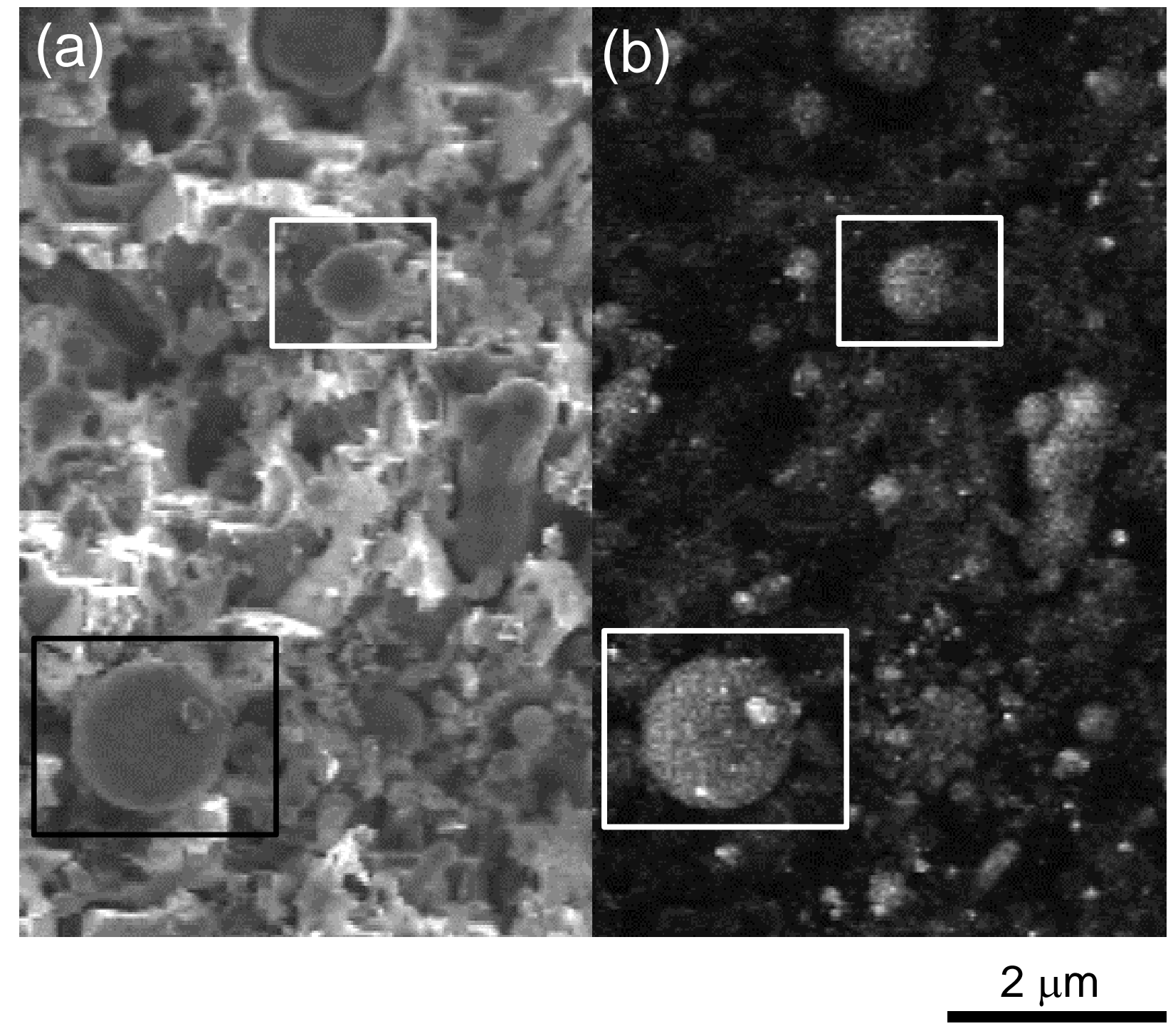

Fig. 7, T. Ohashi et al. 


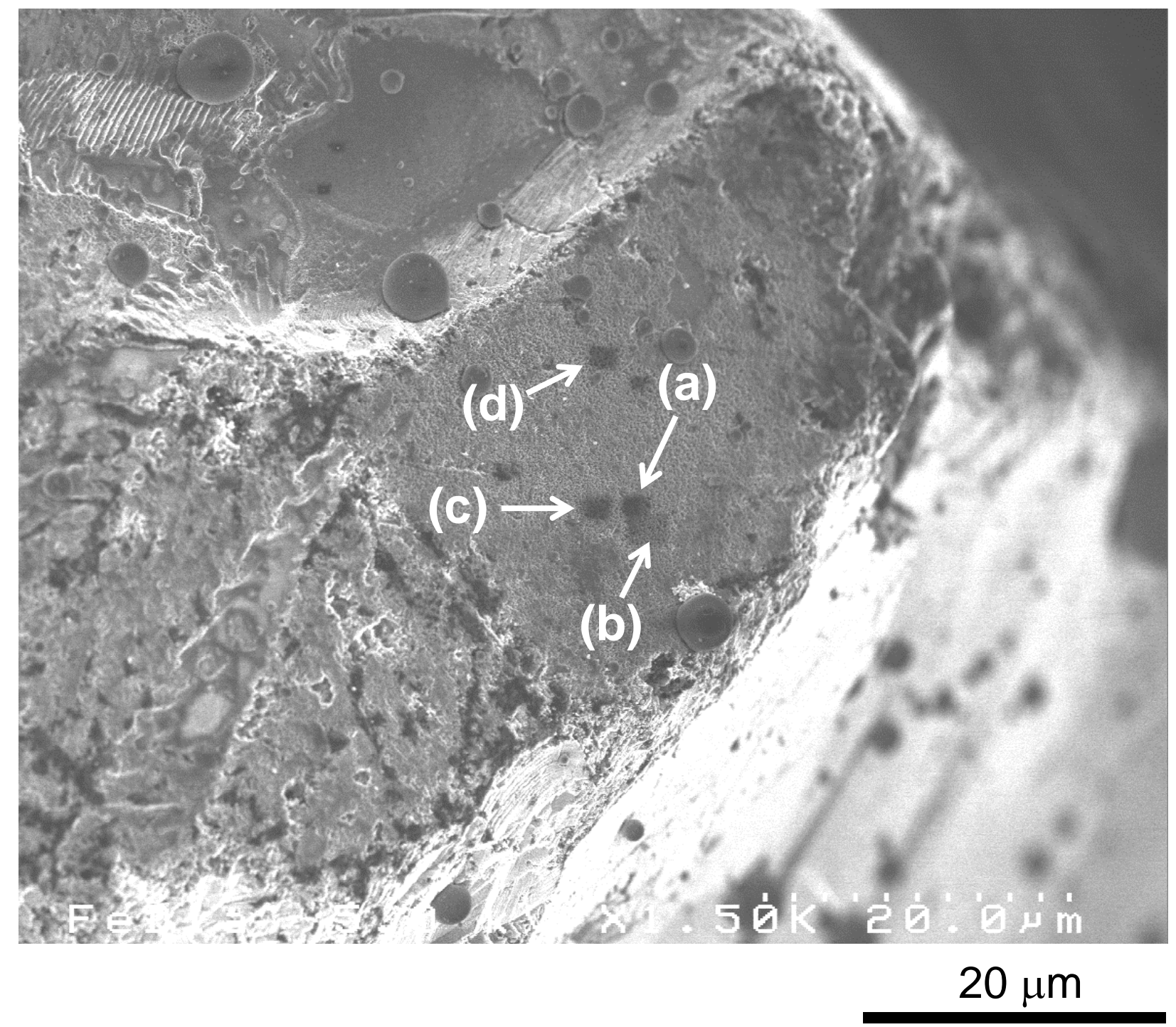

Fig.8, T. Ohashi et al. 

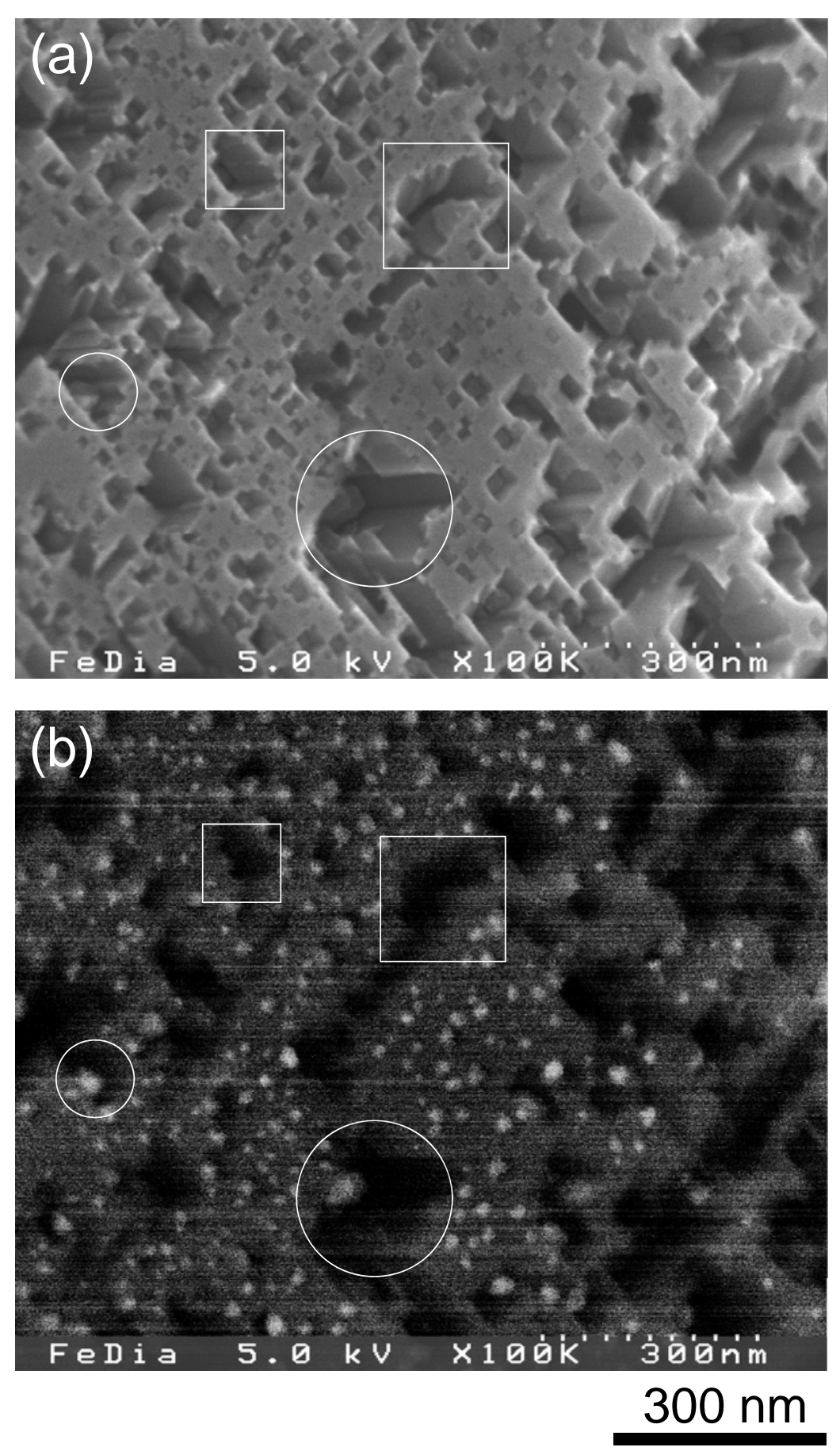

Fig. 9, T. Ohashi et al. 

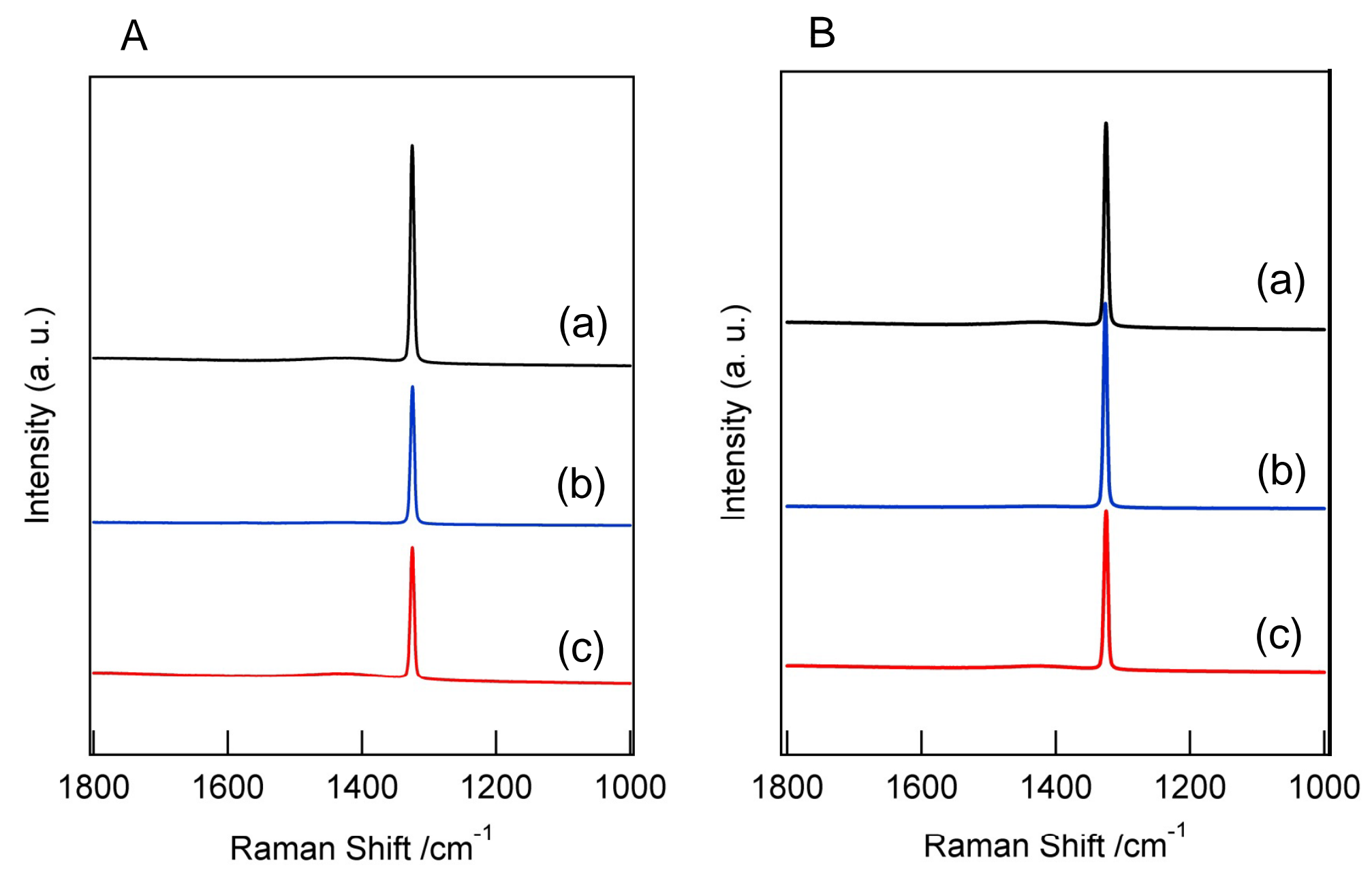

Fig. 10, T. Ohashi et al. 